\title{
Final Report: Sensorpedia Phases 1 and 2
}


This material is based upon work supported by the U.S. Department of Homeland Security under U.S. Department of Energy Interagency Agreement 43WT10301. The views and conclusions contained in this document are those of the authors and should not be interpreted as necessarily representing the official policies, either expressed or implied, of the U.S. Department of Homeland Security. 


\title{
SERRI Project: Sensorpedia
}

\section{Final Report: Sensorpedia Phases 1 and 2}

\author{
Bryan Gorman and David Resseguie
}

Date Published:

May 2010

\section{Prepared for}

U.S. Department of Homeland Security

under U.S. Department of Energy Interagency Agreement 43WT10301

Prepared by

OAK RIDGE NATIONAL LABORATORY

Oak Ridge, Tennessee 37831-6283

managed by

UT-BATTELLE, LLC

for the

U.S. DEPARTMENT OF ENERGY

under contract DE-AC05-00OR22725 



\section{CONTENTS}

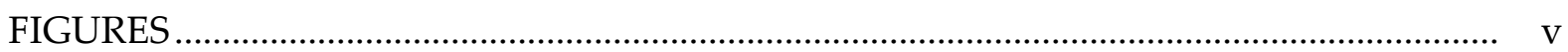

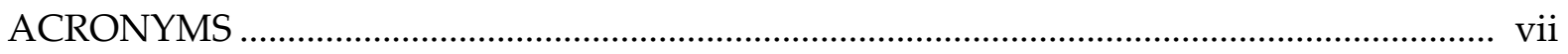

SOUTHEAST REGION RESEARCH INITIATIVE .......................................................... ix



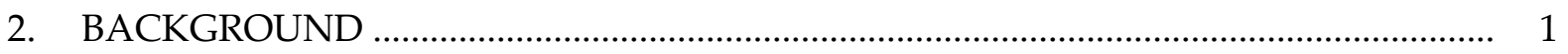

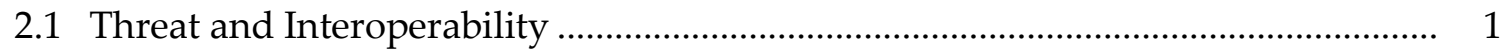

2.2 The Internet and Government........................................................................... 1

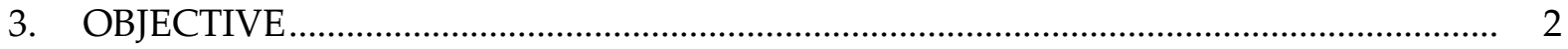

3.1 What is Sensorpedia? ........................................................................................... 2

3.2 What Kind of Sensors Will Connect to Sensorpedia? .............................................. 2

3.3 Who Will Use Sensorpedia? ................................................................................... 3

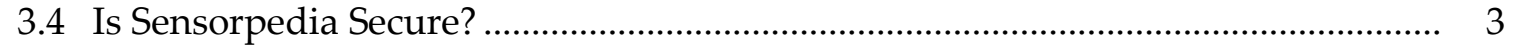

3.5 How Will Sensors Connect to Sensorpedia? ............................................................ 3

3.6 What Are the Potential Applications for Sensorpedia? ........................................... 3

3.7 How Much Will Sensorpedia Cost? ....................................................................... 4

3.8 Is There Technical Documentation for Sensorpedia? ............................................. 4

3.9 When Will Sensorpedia Be Ready? ........................................................................... 4

4. OVERVIEW OF SENSORPEDIA BETA …................................................................. 4

4.1 Sensorpedia Beta and Collaborators ........................................................................ 4

4.2 Sensorpedia: The Software …................................................................................ 4

4.2.1 Sensorpedia Web Application .................................................................. 5

4.2.2 The Sensorpedia Web Services Interface...................................................... 6

4.2.3 The Atom Framework ............................................................................. 7

4.3 Sensorpedia on Social Networks ........................................................................ 8

5. SENSORPEDIA BETA RESULTS ......................................................................... 9

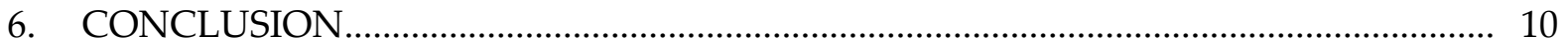

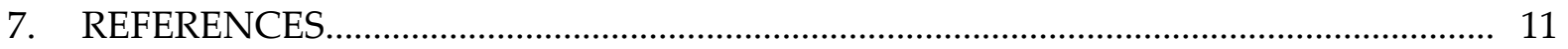

APPENDIXES

Appendix A. Technical Progress Milestones ..................................................................

Appendix B. Program Presentations, Meetings, and Demonstrations .......................... B-1 



\section{FIGURES}

1. Sensorpedia screenshots .............................................................................................. 6

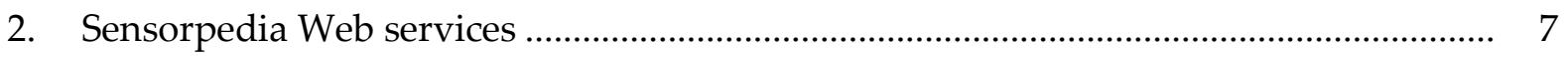

3. Sensorpedia software architecture …………………………………………………. 8

4. Sensorpedia tag cloud ........................................................................................... 9

5. Sensorpedia applications suggested by the beta test team ................................................ 10 



\section{ACRONYMS}

AOF

API

CDC

CTS

DHS

DoD

DOE

DTRA

GA

GeoRSS

IC

KML

MSSI

NGA

NOAA

ORNL

RESTful

RSS

SensorML

SERRI

SOFIC

UT

WFS activity, objects, and features

application programming interface

Centers for Disease Control and Prevention

Collaborative Technologies and Systems (international symposium series)

U.S. Department of Homeland Security

U.S. Department of Defense

U.S. Department of Energy

Defense Threat Reduction Agency

general availability

geodata/spatial data combined with RSS

intelligence community

Keyhole Markup Language (Google Earth)

Multi-State Sharing Initiative

National Geospatial-Intelligence Agency (DoD)

National Oceanic and Atmospheric Administration

Oak Ridge National Laboratory

(conforming to the) Reprsentational State Transfer (protocols)

Really Simple Syndication

Sensor Model Language (Open Geospatial Consortium encoding

standard)

Southeast Region Research Initiative

Special Operations Forces Industry Conference

University of Tennessee

Web Feature Service (Open Geospatial Consortium interface standard) 



\section{SOUTHEAST REGION RESEARCH INITIATIVE}

In 2006, the U.S. Department of Homeland Security commissioned UT-Battelle at the Oak Ridge National Laboratory (ORNL) to establish and manage a program to develop regional systems and solutions to address homeland security issues that can have national implications. The project, called the Southeast Region Research Initiative (SERRI), is intended to combine science and technology with validated operational approaches to address regionally unique requirements and suggest regional solutions with potential national implications. As a principal activity, SERRI will sponsor university research directed toward important homeland security problems of regional and national interest.

SERRI's regional approach capitalizes on the inherent power resident in the southeastern United States. The project partners, ORNL, the Y-12 National Security Complex, the Savannah River National Laboratory, and a host of regional research universities and industrial partners, are all tightly linked to the full spectrum of regional and national research universities and organizations, thus providing a gateway to cuttingedge science and technology unmatched by any other homeland security organization.

As part of its mission, SERRI supports technology transfer and implementation of innovations based upon SERRI-sponsored research to ensure research results are transitioned to useful products and services available to homeland security responders and practitioners.

For more information on SERRI, go to the SERRI Web site: www.serri.org. 



\section{INTRODUCTION}

This report is a summary of Oak Ridge National Laboratory's (ORNL's) Phase 1 and 2 progress in the development of Sensorpedia, a sensor information sharing platform. Sensorpedia is ORNL's “Wikipedia for Sensors." Currently in Phase 3 limited beta testing, Sensorpedia will enable global scale sensor information sharing for scientific research, national security and defense, public health and safety, emergency preparedness and response, and general community awareness and outreach once it is in general availability (GA).

\section{BACKGROUND}

\subsection{Threat and Interoperability}

With the growing prevalence of sensor and surveillance networks, a means of sharing sensor data has become a priority of first responders, emergency planners, and disaster managers. In its May 4, 2007, Information Sharing Strategy statement, the U.S. Department of Defense (DoD) offered a vision of an environment that supports "transparent, open, agile, timely, and relevant information sharing ... across a trusted information environment." In command and control, whether for military operations, homeland security, public safety, or emergency response, there is a generally accepted principle that information sharing can significantly enhance situation awareness. At the same time, there is an equally acknowledged criticism that the nation's various emergency response, intelligence, and law enforcement communities lack sufficient interoperability to permit them access to the information they need to connect the dots to anticipate or detect a threat.

\subsection{The Internet and Government}

Today, without any abatement in sight, the Internet continues to expand to include nearinstantaneous information from and about the measurable natural environment and from and about human networks for transportation, communication, energy, commerce, and banking. This networked information is accessible practically anywhere at any time on the millions (and potentially billions) of Internet-enabled mobile phones that are proliferating at unprecedented rates and are themselves producing staggering amounts and varieties of textual, visual, audible, and locational data on social networks that host millions of users. The availability and accessibility of data over the Internet has greatly enhanced the ability of researchers and developers to build applications, conduct analyses, and perform research, and as evidenced by the U.S. Government's various "Gov 2.0" initiatives, sharing information is emerging as a potential strategy for improving organizational efficiency and effectiveness, particularly of large and complex organizations and institutions.

Given the means to search, correlate, and analyze real-time sensor data sources, organizations within the U.S. Department of Homeland Security (DHS) could significantly improve their ability to detect, interdict, mitigate, and respond to emerging trends and threats. 
To this end, DHS, the intelligence community (IC), and DoD have begun to adopt online information sharing capabilities (e.g., wikis, blogs, tags, geotags, and mashups) and catalogs (e.g., the DoD Metadata Registry, UCore, and the National Information Exchange Model) to help connect the dots of organizational information. The IC has shown that it is possible to run a social media Web site securely (the Intellipedia wiki on the Joint Worldwide Intelligence Communications System, the Secret Internet Protocol Router Network, and the Intelink-U run the same MediaWiki software that runs the Internet's Wikipedia). However, while these Gov 2.0 examples are instructive, the current Internet models for information sharing lack at least two critical components for improving organizational situation awareness. First and foremost, a proactive facility is needed to connect the dots between networked datasets and to then alert the key personnel seeking and needing information. Without such a facility, an information sharing environment does not necessarily enhance awareness - it is a data store or cloud with potential connections. Second, cataloging efforts notwithstanding, the types of data that federal users and their applications will need in any given scenario may not, and probably will not, conform to preestablished formats or data standards; therefore, interfacing these data to various applications on an ad hoc basis will be problematic. Moreover, information from varied sources and in varied forms will complicate a "one-over-all" universal search function.

\section{OBJECTIVE}

Over the past several years, ORNL has been actively involved in research to formalize the engineering principles and best practices behind emerging social media and social networking concepts to solve real-time data sharing problems for national security and defense, public health and safety, environmental and infrastructure awareness, and disaster preparedness and response. Sensorpedia, an ORNL Web site, is a practical application of several key social media principles. Dubbed the "Wikipedia for sensors," Sensorpedia is currently in limited beta testing and was selected in 2009 by Federal Computer Week as one of the government's "top 10 social networking sites."

\subsection{What is Sensorpedia?}

Sensorpedia is a Web site like Facebook or Twitter; however, instead of creating a social network to connect people to people, Sensorpedia creates a human-machine network to connect sensors with users and applications. Sensorpedia permits its users to publish, subscribe to, search for, connect to, and view all types of sensor information and to connect to or search for particular sensors/sensor types.

\subsection{What Kind of Sensors Will Connect to Sensorpedia?}

Sensorpedia is "sensor agnostic." By that, we mean that all kinds of sensors will connect to Sensorpedia, including sensors that some argue are not true sensors (e.g., human beings, cell phones, and video cameras). Of course, all kinds of classical sensors (e.g., smoke detectors, intrusion alarms, weather sensors, global positioning systems, seismic sensors, acoustic sensors, chemical sensors, radiological sensors, pressure gauges, medical instrumentation, telemetry systems, home security systems, and alarms) will connect to 
Sensorpedia. There is no conceivable limit to the kinds of sensors and detectors that will connect to Sensorpedia. Even sensor databases can connect with Sensorpedia.

\subsection{Who Will Use Sensorpedia?}

Sensorpedia will serve a broad range of users, from casual one-time users who will search for sensor information from a particular location to scientists, developers, students, and hobbyists who routinely will use Sensorpedia to contribute and retrieve sensor information for experiments and science projects. Local, state, and federal public safety officials, intelligence analysts and planners, and emergency response workers, everyone and anyone with sensor information to share, can use Sensorpedia for improved awareness, planning, security, and emergency response. Even homeowners and business owners who intend to improve the security and energy efficiency of their homes and businesses can use Sensorpedia. What Wikipedia is for general knowledge and information, Sensorpedia will be for sensor information.

\subsection{Is Sensorpedia Secure?}

The security for the Sensorpedia limited beta test is provided by controlled network access to a firewall-protected server and password-protected user login to the application. The open beta version, when it becomes available, will be able to support read and write access permissions based on a user's preferences for his/her approved social network. Alternatively, the Sensorpedia server software can be hosted on an enterprise server and isolated from the Internet to provide secure internal sensor information sharing for specific enterprise use cases where governance of the data and sensors requires isolation from the Internet.

\subsection{How Will Sensors Connect to Sensorpedia?}

In the Sensorpedia limited beta test, an application programming interface (API) provides the software instructions for interfacing sensors, sensor data, and sensor alerts to Sensorpedia. In future versions of the system, adding and sharing sensors will be as easy as linking YouTube videos to Facebook or posting news updates to LinkedIn.

\subsection{What Are the Potential Applications for Sensorpedia?}

In addition to easing scientific, educational, and recreational access to sensor information, Sensorpedia will eliminate a significant amount of system integration for enterprise and public safety applications and permit a trusted social network of collaborators, operators, and users to create, share, and view spatial and temporal "mashups" of multiple sensor sources. Sensorpedia virtually eliminates the need for a tightly coupled system to integrate all the various sensors into a monolithic, homogeneous whole. By easing programmatic access to sensor data, Sensorpedia will simplify the development of sensor fusion and mining applications, as well as the development of new predictive models and simulations, enabling a new generation of sensor applications. 


\subsection{How Much Will Sensorpedia Cost?}

Access and use of an open version of Sensorpedia (i.e., www.sensorpedia.com and www.sensorpedia.org) is intended to remain free for Internet users. For users who have special requirements to run enterprise versions of Sensorpedia inside their firewalls, a licensing plan will be negotiated.

\subsection{Is There Technical Documentation for Sensorpedia?}

Complete documentation of the Sensorpedia API and Web application is available online at http://sites.google.com/site/sensorpedia/.

\subsection{When Will Sensorpedia Be Ready?}

Sensorpedia is currently in a limited beta test release. Phase 3 development, which is underway, will lead to an open beta version in October 2010. A GA version is expected for early 2011.

\section{OVERVIEW OF SENSORPEDIA BETA}

\subsection{Sensorpedia Beta and Collaborators}

Since August 2008, the Southeast Region Research Initiative has funded two completed phases of Sensorpedia development. To launch a limited beta test of Sensorpedia, seven collaborators were selected for an initial release. These collaborators constituted a good cross-section of interests: academic and scientific research, DoD security, public safety and public health, enterprise and international sensor standards development, and even bee husbandry. Their sensors and observations also covered a range of phenomena from weather and environmental effects to space-borne and airborne remotely sensed imagery.

The Sensorpedia limited beta test collaborators and their research interests are as follows.

- Open Geospatial Consortium, Inc.: Sensor Web Standards

- Northrop Grumman Corporation: Interface to PULSENet

- National Oceanic and Atmospheric Administration (NOAA): Weather Sensors

- University of Tennessee: Interface to Robots

- Rochester Institute of Technology: iPhone and Python Library

- Illinois College: Regional Sensors

- Tuskegee University: Air and Water Sensor

\subsection{Sensorpedia: The Software}

Sensorpedia is designed to support a wide variety of users. The core Sensorpedia components have been made available to the academic and research communities to allow easy publication and sharing of sensor data. The use of data portability standards enables the integration of available sensor data into other Web based software applications 
(developed separately from this effort) to support specific communities of interest such as first responders and public health and law enforcement organizations.

Sensorpedia development is divided into two main efforts: a Web based application and the supporting Web services interface. Both efforts leverage proven design concepts and best practices of successful Web 2.0 and social networking Web sites. Complete technical documentation of the Sensorpedia interfaces, including the Atom framework, is at the aforementioned Google site (http://sites.google.com/site/sensorpedia/).

\subsubsection{Sensorpedia Web Application}

The Sensorpedia Web application (which ORNL has deployed in a limited beta release as of March 2009) provides a browser-based interface to explore, contribute to, and share data from online sensor systems. The online beta site is available at http://www.sensorpedia.com. The application's design has as its priority an intuitive interface for registering sensors, searching for relevant data, and subscribing to sensor observations. The Sensorpedia application provides common Web 2.0 features such as tagging, mashups, inline editing, and a recommendation engine. The application also provides social networking features that allow users to establish trusted groups, share relevant views and sensor data, and even publish updates to external applications such as LinkedIn, Facebook, and Twitter.

In terms of its underlying design, the Sensorpedia application follows a methodology that Joshua Porter describes in his book, Designing for the Social Web [Porter, 2008]. Porter's design prioritization scheme is drawn from his research into the commonalities of highly successful social Web applications. In understanding the commonalities of the sites he studied, Porter derived a methodology for characterizing, comparing, and contrasting their underlying designs. He termed his methodology the AOF Method for the three discrete elements of the Web sites: activities, objects, and features.

In the AOF Method, the primary element of a successful social media application is its focus on a specific activity. For example, YouTube's primary activity is sharing videos. Facebook's primary activity is connecting (or reconnecting) its members with family and friends. Wikipedia's core activity is the collaboration on an online encyclopedia.

In the AOF Method, application activities are centered on social objects, the nouns of social Web design. These objects may correspond to real-life items or they may be abstractions. For Flickr, a popular photo sharing activity, the social objects are the photographs themselves. Job postings are the social objects of job search sites like Monster.com and CareerBuilder.com. Social objects provide the connection between users in a social network. A key to success for many popular applications has been the assignment of a unique URL to each social object. URLs make objects easy to bookmark, share, and embed in other applications.

Along with the primary activity and social objects, the core features of an application are defined as the set of possible actions a user can take on each object. The features are the verbs associated with the nouns. For example, core features for online shopping sites like Amazon include purchasing an item, writing product reviews, and adding items to a wish list.

The AOF Method informed and focused the formal design of Sensorpedia. Sensorpedia's primary activity is sensor data sharing. Its social objects are the sensors and associated observation data. Each sensor is provided a unique URL to support bookmarking and 
sharing. From these objects, the core feature set provides the functionality to register sensors, establish social networks of trusted collaborators, and explore available sensor data.

The main user interface of the application is a map-based mashup (see Sensorpedia screenshots in Figure 1) that allows for browsing and discovering available sensor data by location and keywords (tags).


Figure 1. Sensorpedia screenshots.

\subsubsection{The Sensorpedia Web Services Interface}

Sensorpedia consists of Web services. The Web services are designed to accept and publish data using popular data and interface standards such as streaming media, Google Earth Keyhole Markup Language, and GeoRSS. Sensorpedia also relies on OpenID for future interoperability with other Web based authentication systems.

The Sensorpedia Web services interface (i.e., API) provides an easy mechanism for interfacing discrete and incompatible sensor data to existing and future enterprise applications (see Figure 2). 




Figure 2. Sensorpedia Web services.

\subsubsection{The Atom Framework}

The Sensorpedia API is based on the Atom syndication format, an XML used for Web feeds. The Atom 1.0 specification was released in December 2005 by the Internet Engineering Task Force. Web feed standards such as Atom and RSS (Really Simple Syndication) were originally developed to provide an automated method to check for updates published on a Web site without requiring a user to visit a site in a browser. Instead, Atom published a list (or "feed") of recent articles or content in a standardized, machine-readable format. Many feed readers and aggregators have been developed to manage feed subscriptions. The Atom content model is flexible and extensible, and the specification was written to support a wide variety of content beyond Web site update notifications.

The Atom model also allows for links to additional resources for visualization, alternate format representations, and any number of other requirements. The Sensorpedia API uses the Atom content model to represent registered sensor systems and to provide subscription links to observation data. Since the Atom specification supports extensions, the Sensorpedia API specifies the GeoRSS format to represent spatial information associated with each 
sensor entry. A simple RESTful Web service API is used to provide insert, update, delete, and query operations on the stored Atom documents (see Figure 3).

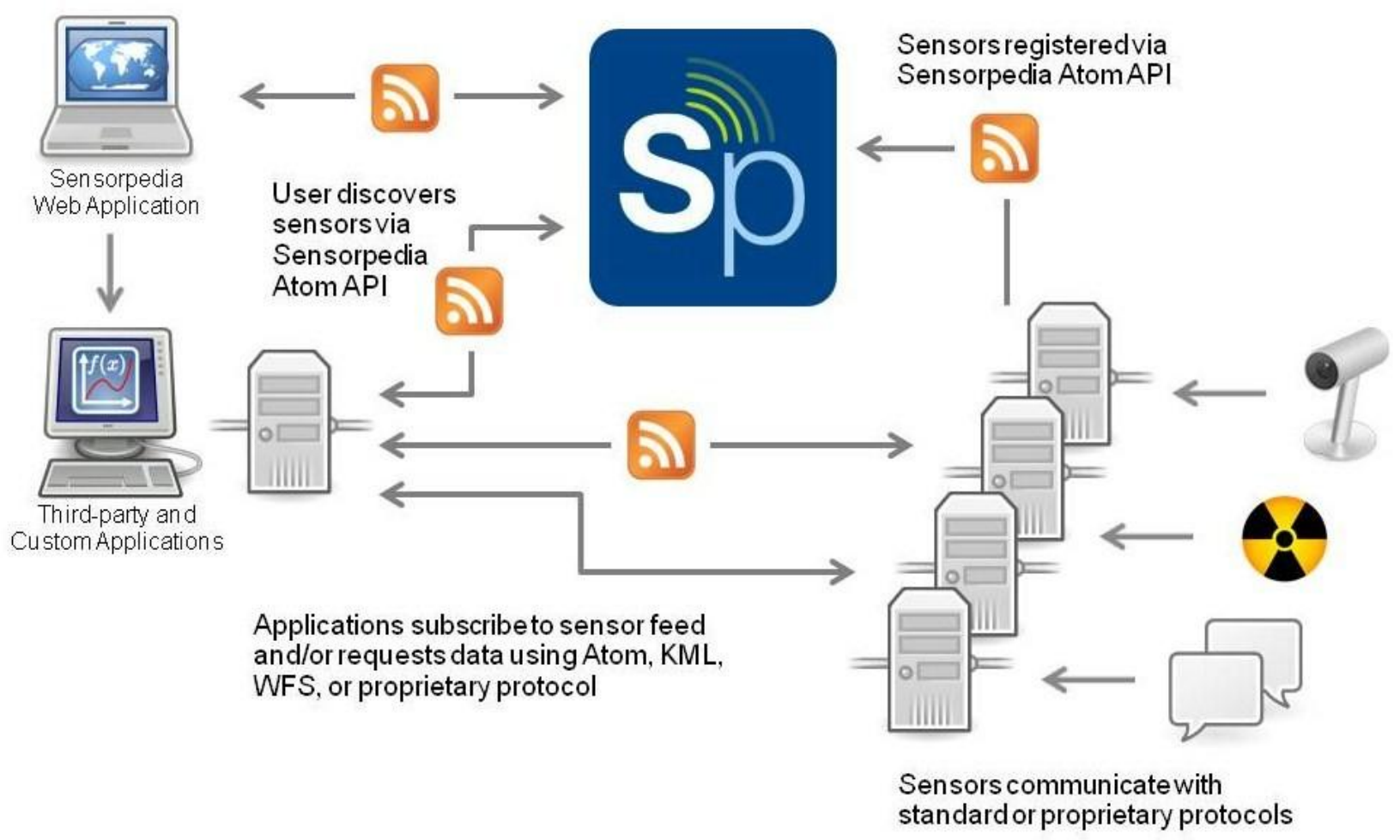

Figure 3. Sensorpedia software architecture.

The Atom based API does not constrain data publishers to a given data format for representing observation data. Rather, the API provides the ability to link to any number of representations of the data, such as Atom, GeoRSS, SensorML, Web Feature Service, Sensor Observation Service, HTML, or other proprietary formats. The Atom format also allows the embedding of observation data within the feed document itself, making the Atom feed self contained.

The Sensorpedia Web service provides the virtual infrastructure to index data from many disparate sources. While the Sensorpedia API provides operations to retrieve Atom documents describing data sources by searching on several fields, the search function is currently limited to matching queries to known values or ranges. This presents a challenge in discovering relevant data among a large number of data sources. Future extensions to Sensorpedia will use collective intelligence algorithms and other artificial intelligence techniques to connect users with potential information sources of interest and will form connections between users based on common informational needs.

\subsection{Sensorpedia on Social Networks}

The Sensorpedia community is present on the following publicly accessible social networks. 
- Sensorpedia Blog (http://www.sensorpedia.org/blog)

- Sensorpedia on Twitter (http://twitter.com/Sensorpedia)

- LinkedIn Sensorpedia Group (http://linkedin.com)

- Sensorpedia Lens on Squidoo (http://squidoo.com/Sensorpedia)

- Sensorpedia Photos on Flickr (http://flickr.com)

\section{SENSORPEDIA BETA RESULTS}

As of March 2010, more than 4,000 sensors worldwide had been indexed by Sensorpedia (see the Sensorpedia tag cloud in Figure 4). Most of the sensors are land and buoy weather sensors in the United States. As the tag cloud shows, much of the information is weather related, coming from International Civil Aviation Organization and NOAA weather stations and buoys. Sensorpedia also indexes a number of traffic cameras from around the southeastern United States, including a concentration of cameras in Charleston, South Carolina (thus it's prominence in the tag cloud). Other important sensor types monitor seismic activity, energy efficiency, and water levels. Then there are the more obscure sensors like those used for monitoring bacteria levels at beaches. The greatest concentration of sensors is still in the United States, but several sensor systems are also included from Europe, Asia, and the Arctic regions.

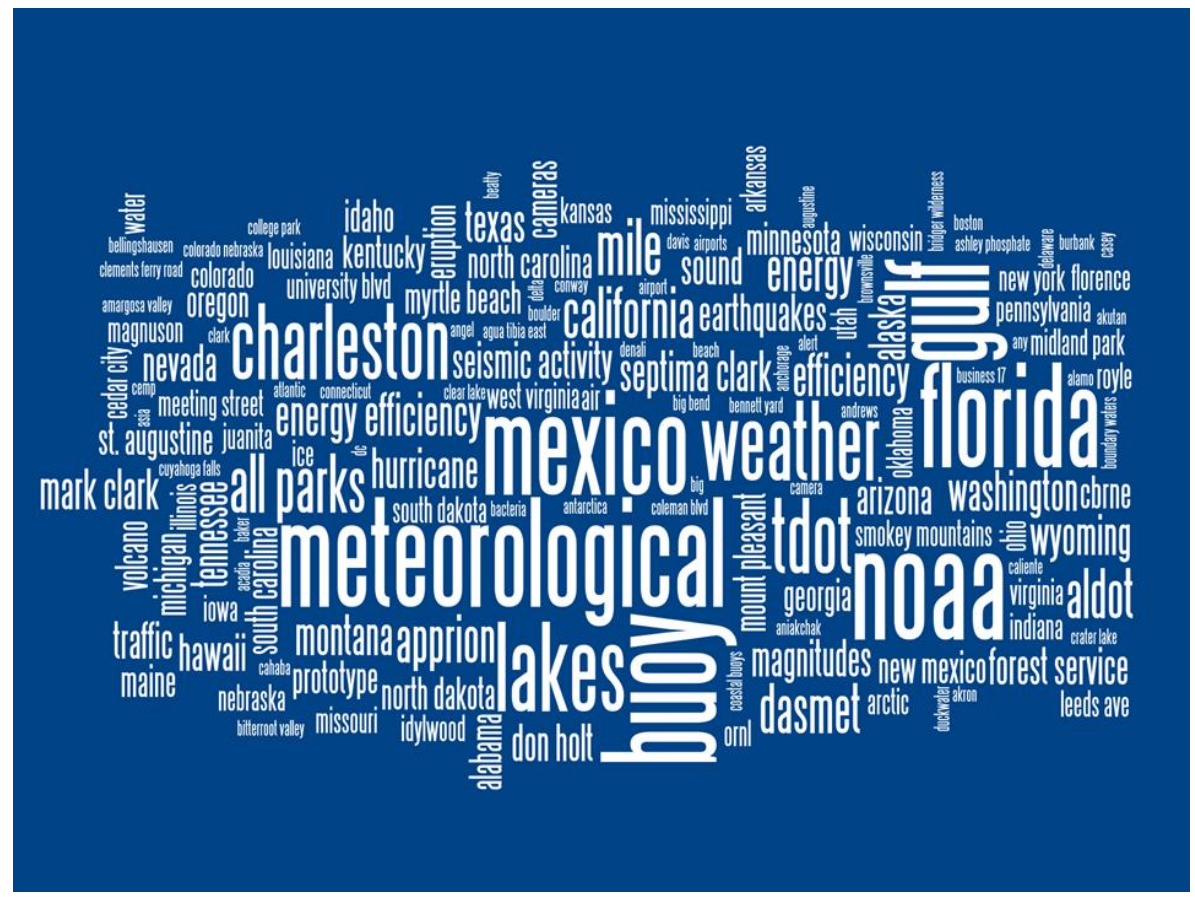

Figure 4. Sensorpedia tag cloud.

Although Sensorpedia is intended to satisfy prevailing needs for sensor information sharing in support of public safety and security requirements, it is also envisioned as an evolutionary platform that will support the next generation of sensor information sharing technologies. For example, it is conceivable that Sensorpedia will in the near future interface 
with a cognitive agent application that will routinely and autonomously act on behalf of human analysts to review anomalistic sensory observations or with a location-aware robotic system that will scan inaccessible or highly vulnerable locations on a $24 / 7$ basis to discover, observe, measure, and post real-time sensor information.

The beta test team provided a number of novel applications for Sensorpedia (see Figure 5), including a Python programming library, an autonomous robot interface, water and air sensor Web sites, and an iPhone application. Detailed descriptions of these applications are provided on the Sensorpedia blog (http://www.sensorpedia.com/blog/).
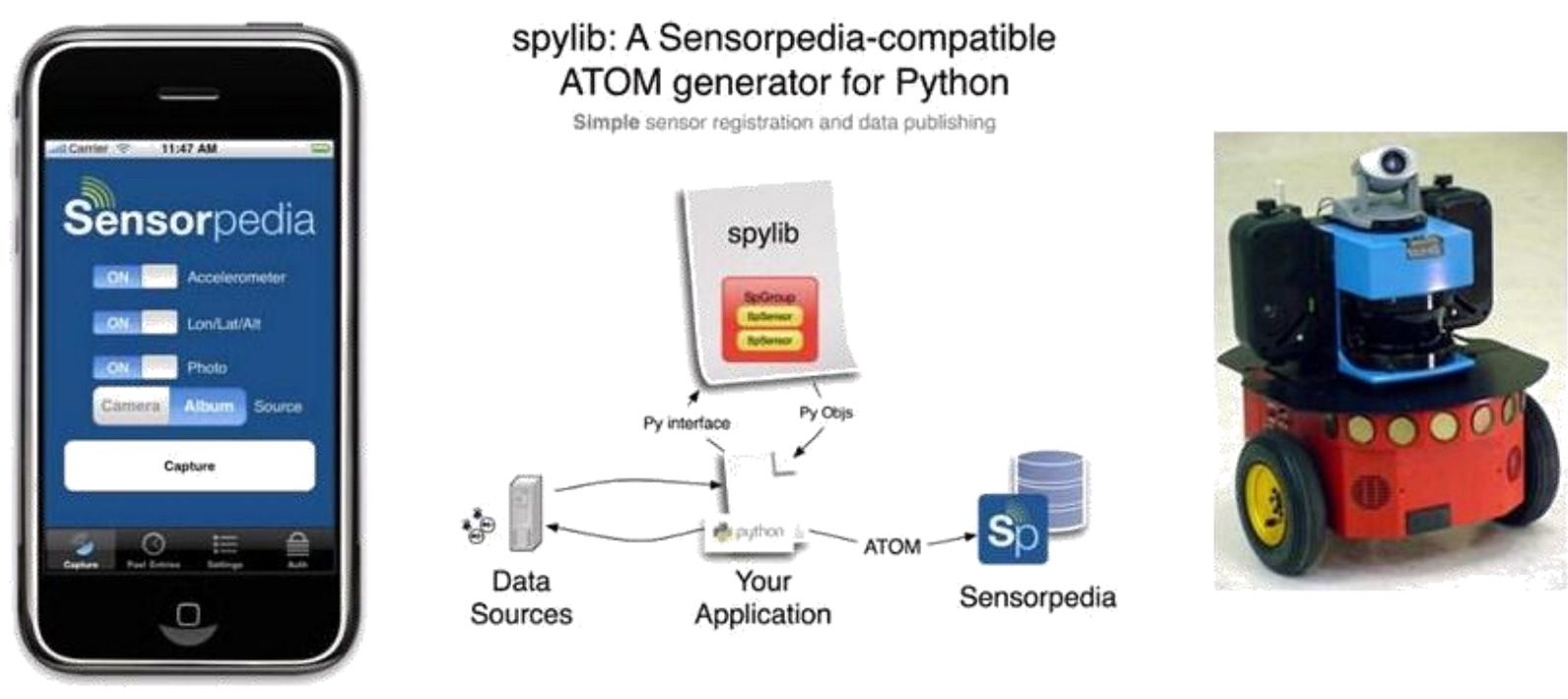

Figure 5. Sensorpedia applications suggested by the beta test team.

\section{CONCLUSION}

The Phase 1 and Phase 2 development of Sensorpedia concluded with a limited beta test that indexed almost 4,000 sensors and led to Sensorpedia's ranking by Federal Computer WEEK as one of the government's top 10 social networking sites. Clearly, the concept for a Wikipedia for Sensors has been successful; however, work remains to make Sensorpedia a tool for homeland security. A social networking component to control access and read/write privileges to sensor information needs to be implemented. The system needs to be tested on a much larger user base with a much larger database of sensors (100 times larger). Phase 3 , which is already underway, will address those requirements. At the conclusion of Phase 3, DHS will be able to bring the Sensorpedia software inside its enterprise, much as the IC has done with Wikipedia, to establish a secure, enterprise friendly version. The Sensorpedia enterprise version will permit a trusted social network of DHS collaborators, operators, and users to create, share, and view spatial and temporal mashups of multiple sensor sources without the need for a tightly coupled system. 


\section{REFERENCES}

Institute of Electrical and Electronics Engineers. IEEE Standard Computer Dictionary: A Compilation of IEEE Standard Computer Glossaries. New York, NY: 1990.

J. Porter. (2008). Designing for the Social Web (Voices That Matter), Berkeley, California: New Riders Press.

B. L. Gorman, D. R. Resseguie, and C. Tomkins-Tinch. (2009). “Sensorpedia: Information sharing across incompatible sensor systems," pp.448-454, 2009 International Symposium on Collaborative Technologies and Systems.

B. L. Gorman, D. R. Resseguie, and M. Shankar. (2009). “Designing Information Interoperability," Special Operations Forces Industry Conference (SOFIC). 





\section{APPENDIXES}







\section{APPENDIX A. TECHNICAL PROGRESS MILESTONES}

\section{December 2008}

- Released prelaunch Sensorpedia.com Web site to promote program and identify initial users

- Began engagement of existing online community through the creation of discussion communities on LinkedIn, Twitter, Squidoo, and Facebook

- Designed and developed database structure and Web service application programming interfaces (APIs)

- Development on track for release of beta Web application in January 2009

January 2009

- Continued development of Sensorpedia Web application towards beta release

- Identified initial beta testers and data to be interfaced to Sensorpedia

- Continued outreach via social networking tools (i.e., blogs, LinkedIn, Flickr)

- Continued design and development of database structure and Web service APIs

February 2009

- Continued development of the Sensorpedia Web services API

- Started the Sensorpedia Atom API description document

- Completed three how-to guides interfacing various sensors to Sensorpedia, including implementation of an Atom feed

- Completed implementation of core explore functionality

- Performed cross-browser testing for Internet Explorer, Firefox, Safari, and Chrome

- Notified 10 testers for the Sensorpedia beta launch

\section{March 2009}

- Completed development of the Sensorpedia Web services API

- Completed the Sensorpedia Atom API description document 


\section{April 2009}

Completed Phase 1 and began Phase 2

Began implementation of the Sensorpedia Web services interface

Continued development of the Sensorpedia Web applications

\section{May 2009}

- Launched Sensorpedia beta Web services and Web application

- Created Sensorpedia beta collaboration site

- Coordinated with internal Oak Ridge National Laboratory (ORNL) beta testers to use Sensorpedia framework and services for 4 internal development efforts

- Continued incorporating beta feedback into Web services and application

- Began design and development of "3rd party" applications to prove Sensorpedia framework

- Began integration of a wide variety of sensors, including weather, traffic, and environmental monitoring

June 2009

- Released spylib, a Python library for programmatically generating Sensorpedia Atom documents

- $\quad$ Sensorpedia has over 3,000 registered sensors as of July 31

- Nearing completion on a Sensorpedia iPhone application that facilitates "humans as sensors"

- Nearing completion of a Web based form to register sensors without requiring knowledge of the Atom framework supporting Sensorpedia

- Atom framework now being used by multiple Southeast Region Research Initiative (SERRI) Multi-State Sharing Initiative (MSSI) projects, Tracking 2.0 applications, and several other ORNL projects and proposals

- Sensorpedia supported demonstration to the U.S. Department of Energy Office of Energy Efficiency and Renewable Energy 


\section{August 2009}

- Sensorpedia has over 3,500 sensors as of August 31, 2009

- Extensive performance tuning and enhancements, reducing typical query times from 2+ minutes to $1-2$ seconds

- Upgraded Sensorpedia database to latest version of PostgreSQL (8.4)

- Modified Web services to support lazy-loading of large data sets

- Atom framework actively being used by several SERRI MSSI projects and Tracking 2.0 applications and has been included in several other ORNL projects and proposals

\section{September 2009}

- Numerous software fixes and performance enhancements based on beta testing (for both Web application and services layer)

- Opened Sensorpedia explore and search functions for public viewing without requiring beta account login

- Added single sensor display functionality to Web application to support "ubiquitous URL" Web 2.0 principle

- Began implementation of social networking functionality by evaluating Google Friend Connect, Facebook Connect, and Twitter Oauth

- Began porting of "Tables" sensor networking application to support manipulation of heterogeneous, distributed sensor data from Sensorpedia in a structured environment

\section{October 2009}

- Began implementation of user profiles using OpenID and Google Friend Connect

- Researched advanced tagging system to enhance Sensorpedia search and classification

- Added additional external beta testers and incorporated feedback

- Started sensor indexing for automatic sensor data identification, collection, and integration with Sensorpedia

- Started Sensorpedia "virtual" scripting layer to facilitate interacting with Tables and other end-user tools 





\section{APPENDIX B. PROGRAM PRESENTATIONS, MEETINGS, AND DEMONSTRATIONS}

\section{December 2008}

- Sensorpedia.com prelaunch Web site received numerous user requests to participate in the beta program

- Participation in presentations, meetings, and online interaction with potential Sensorpedia contributors and users has raised awareness of Web 2.0 and social networking applicability to information sharing

- ALPHA prerelease Sensorpedia software was used in Oak Ridge National Laboratory's (ORNL's) Melton Valley Sensornet demonstration [no cost to Southeast Region Research Initiative (SERRI)] to provide additional proof of concept for Sensorpedia objective

- Significant contacts with U.S. Special Operations Command, Raytheon, U.S. Department of Homeland Security (DHS) Office of Health Affairs, Centers for Disease Control and Prevention (CDC), Mars, and the Defense Threat Reduction Agency (DTRA).

January 2009

- Briefed Dr. Marsha L. Vanderford, Director, Emergency Communication System, and Dr. Jay M. Bernhardt, Director, Coordinating Center for Health Information and Service, CDC

- $\quad$ Briefed Robert Burkhardt, Geospatial Information Officer, U.S. Army

- $\quad$ Briefed Mike Scherr, DTRA

- Briefed the Director of Corporate Strategic Research, Raytheon

- Briefed Dr. Starnes E. Walker, Director of Research, DHS

\section{February 2009}

- Brandon M. Rives and Brandon C. Zachary accepted appointments in the U.S. Department of Energy (DOE), Office of Science, Science Undergraduate Laboratory Internships program at ORNL for the summer. Both students of Tuskegee University worked on the Sensorpedia project

- Demonstrated use of Sensorpedia to a group of DOE national laboratory and Battelle personnel (High-Flux Isotope Reactor demo) 
- Briefed Sensorpedia to Professor Alex Bordetsky and his graduate seminar at the Naval Postgraduate School

- Submitted "Sensorpedia: Information Sharing Across Autonomous Sensor Systems," to the 2009 International Symposium on Collaborative Technologies and Systems (CTS 2009)

\section{March 2009}

- Presented Sensorpedia at the SERRI mid-year review in Washington, D.C.

- The submission of "Sensorpedia: Information Sharing Across Autonomous Sensor Systems," was accepted by CTS 2009

- Submitted a paper, "Designing Information Interoperability," to the 2009 Special Operations Forces Industry Conference (SOFIC'09)

- Met with Colonel Art Clark, Program Manager for DoD Integrated Unit, Base, and Installation Protection to discuss Sensorpedia

\section{April 2009}

- Presented Sensorpedia to Mr. Bear McConnell of the DoE U.S. Northern Command

- Presented Sensorpedia to Dr. Vonna Heaton of the National Geospatial-Intelligence Agency (NGA)

- The submission of "Designing Information Interoperability" was accepted by SOFIC '09

- Presented Sensorpedia at the Gov 2.0 Boot Camp in Knoxville, Tennessee

- Presented Sensorpedia to the DoD National System for Geospatial Intelligence Research and Development Test and Evaluation Forum (NRF 2.0) at ORNL

May 2009

- Met with Information International Associates, Inc., in Oak Ridge to discuss establishing a national Web 2.0 conference for first responders

- Presented "Sensorpedia: Information Sharing Across Incompatible Sensor Systems" at CTS 2009

- Met with Billy Stair, UT-Battelle's Communications \& External Relations Director, to discuss Sensorpedia and ORNL's use of social media. 


\section{June 2009}

- Richard Edwards, Jason Frank, Tim Garvin, Brandon Rives, Darren Shea, Christopher Tomkins-Tinch and Brandon Zachary started their summer internships

- $\quad$ Sensorpedia paper presented at SOFIC ‘09

- Presentation on Sensorpedia provided to visitors from NGA

- Presentation on Sensorpedia provided to Dr. William Michener, University of New Mexico, and candidate for the Governor's Chair at the University of Tennessee (UT)

- Presentation of Sensorpedia to faculty of Florida State University

July 2009

- Interviewed by Kristen Letsinger, a freelance contributor to the Knoxville News Sentinel, for a story on social networking and Sensorpedia

- Hosted a visit from the MIT Auto-ID Lab and Georgia Tech Research Institute and provided a briefing on Sensorpedia

- Provided a Sensorpedia presentation to Mr Matthew Poe of the Office of Naval Research

- Provided a Sensorpedia presentation to Captain David Maynard, Commanding Officer, Office of Naval Research Global

\section{August 2009}

- Presented Sensorpedia and the Atom framework for a Tracking 2.0 presentation at the 2009 DOE Packaging Certification Program Technical Review

- Technical discussions with Dr. Jeff de La Beaujardiere, Integrated Ocean Observing System Program Office, National Oceanic and Atmospheric Administration

- Sensorpedia student interns from Tuskegee University, the Rochester Institute of Technology, and UT participated in ORNL's summer student poster session

- Technical discussions with Roger Hixson, National Emergency Number Association Technical Issues Director

\section{September 2009}

- Presented Sensorpedia and the Atom framework at "Tap the Collective" in Washington, D.C., on September 2, 2009 
- ORNL submitted a proposal to extend Sensorpedia's social networking components to Lockheed Martin's Shared Vision Program

- Presented Sensorpedia to ORNL's Computational Biology and Bioinformatics Group and members of ORNL's leadership team (Thomas Zacharia, Billy Stair, and Herb Debban)

- Presented Sensorpedia to the chief information officiers of the U.S. Department of theTreasury and Office of Naval Research - Global

\section{October 2009}

- Presented Sensorpedia and discussed Web 2.0 and resiliency with the U.S. Pacific Command Center for Excellence in Disaster Management and Humanitarian Assistance

- Discussed Sensorpedia technology transfer with Don Kretz, of Raytheon

- Presented Sensorpedia and technology transfer with representatives of Northrop Grumman Corporation. Specific interest in participating in Sensorpedia/PulesNet collaboration 